\title{
Sensorimotor training in virtual reality: A review
}

\author{
Sergei V. Adamovich ${ }^{\mathrm{a}, \mathrm{b}, *}$, Gerard G. Fluet ${ }^{\mathrm{b}}$, Eugene Tunik ${ }^{\mathrm{b}}$ and Alma S. Merians ${ }^{\mathrm{b}}$ \\ ${ }^{a}$ New Jersey Institute of Technology, Department of Biomedical Engineering, University Heights, Newark, NJ, USA \\ ${ }^{\mathrm{b}}$ University of Medicine and Dentistry of New Jersey, Department of Rehabilitation and Movement Science, \\ Newark, NJ, USA
}

\begin{abstract}
Recent experimental evidence suggests that rapid advancement of virtual reality (VR) technologies has great potential for the development of novel strategies for sensorimotor training in neurorehabilitation. We discuss what the adaptive and engaging virtual environments can provide for massive and intensive sensorimotor stimulation needed to induce brain reorganization. Second, discrepancies between the veridical and virtual feedback can be introduced in VR to facilitate activation of targeted brain networks, which in turn can potentially speed up the recovery process. Here we review the existing experimental evidence regarding the beneficial effects of training in virtual environments on the recovery of function in the areas of gait, upper extremity function and balance, in various patient populations. We also discuss possible mechanisms underlying these effects. We feel that future research in the area of virtual rehabilitation should follow several important paths. Imaging studies to evaluate the effects of sensory manipulation on brain activation patterns and the effect of various training parameters on long term changes in brain function are needed to guide future clinical inquiry. Larger clinical studies are also needed to establish the efficacy of sensorimotor rehabilitation using VR in various clinical populations and most importantly, to identify VR training parameters that are associated with optimal transfer to real-world functional improvements.
\end{abstract}

Keywords: Virtual reality, virtual environment, sensorimotor training, rehabilitation

\section{Introduction}

Virtual reality (VR) can be defined as an approach to user-computer interface that involves real-time simulation of an environment, scenario or activity that allows for user interaction via multiple sensory channels [19]. VR technology, and its application, is rapidly expanding across a variety of disciplines. Virtual environments (VEs) in VR can be used to present richly complex multimodal sensory information to the user and can elicit a substantial feeling of realness and agency, despite its artificial nature [105].

Virtual reality systems are generally classified by the visual presentations they provide to a participant,

*Address for correspondence: Sergei Adamovich PhD, Motor Control and Rehabilitation Laboratory, Department of Biomedical Engineering, New Jersey Institute of Technology, 665 Fenster Hall, University Heights, Newark, NJ 07102, USA. Fax: +1 973596 5222; E-mail: sergei.adamovich@njit.edu. the presence or absence of somatosensory feedback and the modality used to collect data from the participant. Visual stimuli are grouped by the level of immersion. Two-dimensional presentations are considered non-immersive. Three dimensional presentations utilizing stereoscopic projections or displays with a fixed visual perspective are considered semi-immersive. Fully immersive systems allow for changing visual perspective with head movement. There are a myriad of methods of collecting data from a subject. Some systems utilize joysticks, hand controls or steering wheels. Motion tracking systems that utilize video and optoelectronic cameras, electromagnetic and ultrasound sensors, accelerometers and gyroscopes provide kinematic data. Instrumented gloves can add precision to tracking of hand motion. The data collected from these devices is used to control a computerized representation of the user or an avatar that represents their movements and interacts with the VE. Video capture virtual reality 
(VCVR) is a family of video camera based motion capture systems that record and digitize pictures of participants as they move, and transfer those images into a virtual environment, in real time [123]. These systems differ from other forms of VR in terms of their visual presentation which is a mirror image of the participant. Flicker glasses that display alternating right/left views of the picture or head-mounted visual displays (HMD) may be used for greater immersion (for both gait and upper extremity systems). The most immersive system is the CAVE (University of Illinois at Chicago) which is a room-size, 3D video and auditory system. Finally, newer systems that utilize robots to provide interaction forces between the user and VE are classified as haptic systems. Several systems like GENTLE-S [27], MITManus [1] and PneuWREX [127] can be used to provide haptic effects during upper extremity activities in VEs. LOKOMAT (Hocoma) is a robotic exoskeleton, while CAREN System (Motek) utilizes self-paced motorized treadmills mounted on a 6 degree-of-freedom motion. These systems are designed to facilitate gait training, and both systems can be integrated with VE by presenting virtual locomotion scenarios displayed on a screen in front of the subject. The CAREN system can be combined with a harness for safety or body weight support.

Many disciplines of healthcare now rely on VR, such as for training surgeons [80], delivery of cognitive therapy [102], and delivery of post-traumatic stress disorder therapy [108]. The use of VR for sensorimotor training is a promising addition to its already broad utility in healthcare. Initial investigations into this family of approaches to rehabilitation emerged in the mid 1990 's. Several reviews summarize the first generation of this research $[52,63,107,117,123]$, with more recent systematic reviews examining the clinical efficacy of sensorimotor training in VE for rehabilitating upper extremity function [49] and gait [29] after stroke. This review adds to the existing literature by integrating the above studies with more recent reports, some emanating from our laboratories, that discuss how spatial and temporal manipulations in VR may be used intelligently to enhance sensorimotor training, and how they can be interfaced with robotics for rehabilitation purposes. We conclude with a discussion of the development and efficacy of telerehabilitation applications, which can be interfaced with VR to improve the accessibility of VR to the broader patient population.

\section{Why might training in VR be beneficial for restoring neural function?}

Recent studies show evidence of the potential of VRbased interventions to benefit patients with disordered movement due to neurological dysfunction. Known neurophysiological and behavioral benefits of movement observation $[8,18,25]$, imagery [20], repetitive massed practice and imitation therapies [42] in facilitating voluntary production of movement can be easily incorporated into VR to optimize the training experience and allow the clinician to use sensory stimulation through VR as a tool to facilitate targeted brain networks, such as the motor areas, critical for neural and functional recovery. The potential for functional recovery can be optimized by tapping into a number of neurophysiological processes that occur after a brain lesion, such as enhanced potential for neuroplastic changes early in the recovery phase and stimulation of sensorimotor areas that may otherwise undergo deterioration due to disuse. VR may be useful in a number of ways to deal with these processes and potentially trigger compensatory neuroplastic changes.

\subsection{Mass practice}

Animal and human studies have shown that important variables in learning and relearning motor skills and in changing neural architecture are the quantity, duration and intensity of training sessions. There is evidence to demonstrate that plasticity is "use-dependent" and intensive massed and repeated practice may be necessary to modify neural organization $[57,87,88,118$, 120,128]. The importance of intensity and repetition has also been confirmed for stroke patients in the chronic phase [119] in the treatment paradigm referred to as constraint-induced-movement-therapy (CIMT). Use-dependent cortical expansion has been shown up to 6 months after 12-days of CIMT therapy in people post stroke [78]. Dependence on existing therapies alone to promote neuroplastic changes might not always be practical. For example, changes at the synaptic level are evident in the rodent brain after the animal is exposed to thousands of repetitions of a given task over a short interval of time, i.e. 12,000 repetitions over 2-3 days $[88,101]$. In stark contrast, the affected extremity is moved at best 1-2 hours/day in the weeks after stroke $[10,11]$ and as few as 10-20 repetitions per training session in the chronic phase [74]. More than 50\% of this time is spent on rehabilitating the lower extremities and balance rather than the hand [58-60]. Use of 
VR as a training environment may provide a rehabilitation tool that can be used to exploit the nervous systems' capacity for sensorimotor adaptation by providing a technological method for individualized intensive and repetitive training.

\subsection{Dynamic and operant conditioning of skill}

In addition to the training intensity and volume necessary to induce neural plasticity, sensorimotor stimulation must involve the learning of new motor skills. Empirical data strongly emphasize that learning new motor skills is essential for inducing functional plasticity $[68,89,101]$, therefore, it appears that critical variables necessary to promote functional changes and neural plasticity are the dynamic and adaptive development and formation of new motor skills. It is believed that adaptive training paradigms that continually and interactively move the subject's performance toward the targeted skill are important to optimize re-learning of motor skills [79]. Once again, VR-based applications can provide adaptive learning algorithms and graded rehabilitation activities that can be methodically manipulated to meet this need.

\subsection{VR delivered during critical periods to augment neuroplastic changes}

One of the central problems facing patients and clinicians is that most interventions are impractical to deliver to patients at perhaps the most opportune time, that is during the acute phase of stroke when the potential to harness neuroplastic changes is greatest but during which phase the patient is too paretic to perform hand training. If the same principle that is apparent in the developing nervous system of cats applies to the lesioned adult cortex of humans, that lack of stimulation to motor cortex during a critical period leads to lost corticospinal synaptic connections [36] and that stimulation of motor cortical networks during the same critical period can reinstate some of these otherwise lost connections [109], then the acute paretic phase in stroke may perpetuate functional and neural deterioration simply due to absence of cortical stimulation.

The above sections provide an overview of the multifaceted components in skill re-acquisition, such as mass practice, rich environments, and timing of VR delivery that may mediate neuroplasticity following a lesion. The versatility of VR in these respects offers the clinician various ways to modulate brain reorganization. However, perhaps an even more appealing aspect of VR is its versatility in presenting complex sensory stimulation, through a combination of visual, somatosensory (haptic), and auditory feedback. Intelligent manipulation of these parameters may offer the clinician a yet unattained level of control over the therapeutic efficacy of a given intervention. The current state of the art in using these approaches is reviewed below.

\section{Effects of visual augmentation on neural circuits}

In order to understand the potential of VR to benefit recovery at the functional outcomes level in patient populations, one needs to understand the neural processes that VR may be affecting and, in the case of patient populations, how VR may affect recovery at the neural level. A related and equally important question is whether interacting in VR engages similar neural circuits to those recruited for actions performed in the real-world.

The "wiring" of the brain lends nicely to using visual feedback in VR to augment distributed, but interconnected, cortical regions. For example, retrograde tracer studies show rich intra-hemispheric cortico-cortical connections that link occipital, parietal, and frontal cortices [76,77,113]. Moreover, single unit recordings demonstrate that a substantial number of motor, premotor, and parietal neurons are modulated by visual information [45-47,62], suggesting that visual information can provide a potent signal for reorganization of sensorimotor circuits. At the behavioral level, movement errors in the visual domain can influence motor cortical areas during motor learning $[15,48,84,85,104]$ and active / rewarded practice, can be used to reduce movement errors through feedback, and can shape neural activity in motor and premotor areas $[15,126]$. Finally, even observation of actions (images and video clips), if performed repetitiously and intentionally, can facilitate the magnitude of motor evoked potentials (MEPs) and influence corticocortical interactions (both, intracortical facilitation and inhibition) in the motor and premotor areas $[75,93,112,115]$. This work provides a strong foundation for testing hypotheses on the possible effects that can be produced through visual feedback in VR and opens possibilities for clinical application.

An important consideration in the use of VR as a sensorimotor training tool is the quality of VE rendering compared to what we are used to perceiving in the natural world. In other words, the fidelity of the VR environment may be an important factor in its effec- 
tiveness to recruit neural circuits and deliver desirable outcomes at the functional level. Although VE can be used to provide sophisticated visual information to users and elicit a feeling of real presence [105,106], some work suggests that observation of actions performed by virtual effectors (i.e. the hand) may be less effective in recruiting neural circuits than observation of real hand actions [95]. In a study by Perani, the authors used fMRI to measure the blood oxygen leveldependent (BOLD) signal as subjects observed highfidelity and low-fidelity renderings of a virtual hand perform a reaching task and compared these conditions with a control in which subjects observed real hand actions. The authors found that both virtual conditions produced significantly smaller activation in the frontoparietal circuit that was recruited in the 'real' condition.

However, other evidence suggests that sensorimotor training in VR may actually have similar effects to those noted after real-world training. This evidence comes from several domains. First, studies that have compared the kinematics of movements performed during interaction in a virtual visual environment to those when acting in the real world have found remarkable similarities. For example, healthy subjects responding to targets moving at different velocities exhibit similar movement time, path curvature time, time to peak velocity, and reactions times whether the task is performed in a VE or in the real world [33]. Interestingly, stroke patients' kinematics for reach-to-grasp movements also show similarities in peak wrist velocity, angular shoulder/elbow relationship and maximum grip aperture when acting in the virtual versus a real environment [122].

In two studies performed on persons with stroke, functional improvements following VR training were paralleled by a shift from a predominantly contralesional sensorimotor activation pre-therapy to a predominantly ipsilesional activation post-therapy [54,129]. Similar shifts in hemispheric lateralization are observed after therapy performed in the real world [24,111], suggesting that training an affected limb in VR may tap into similar neural reorganization processes as observed after training in the real world.

Our own work extends this by demonstrating that interacting with virtual representations of ones own hands in VR recruits brain regions involved in attribution of agency [6]. To study such brain-behavior interactions, we integrated our VR system with fMRI and asked thirteen healthy subjects to observe, with the intent to imitate, finger sequences performed by the virtual hand avatar seen in 1st person perspective and animated by pre-recorded kinematic data. These blocks were interleaved with rest periods during which subjects viewed static virtual hand avatars, control trials in which the avatars were replaced with moving non-anthropomorphic objects, or with blocks in which subjects imitated the finger sequence under the above feedback condition. Our data showed a time-variant increase in activation in the left insular cortex for the "observe with the intent to imitate" condition but not in the other conditions. Moreover, imitation with veridical feedback from the virtual avatar (relative to the control condition) recruited the angular gyrus, precuneus, and extrastriate body area, regions which are (along with insular cortex) associated with the sense of agency [4]. Thus, the virtual hand avatars may be useful for sensorimotor training by serving as disembodied tools when observing actions and as embodied "extensions" of the subject's own body (pseudo-tools) when practicing the actions.

The above data inform of potentially useful applications of visual manipulation in VR. For example, intentional observation of movement can be used to stimulate the sensorimotor system without necessitating overt movement itself. Adding more sophisticated manipulations in VR, such as to the color/brightness of objects, their location, form, perspective (1st versus 3rd person), temporal/spatial distortions of the movement trajectory, and feedback replays, can perhaps potentiate these effects in ways that cannot be achieved in the natural world. For example, simulating forward motion by using an optic flow field, and manipulating the speed of the illusory motion during gait training in stroke patients, one can facilitate either faster or slower walking speeds [73].

Another example of a sophisticated VR-based manipulation emerges from an intervention called mirror visual feedback therapy, introduced by Ramachandran and coworkers [7] for amputee and stroke patients. We developed a virtual mirror feedback interface and have used it in conjunction with fMRI to study the effects of this form of visual feedback on neural circuits. In our study [82], a stroke patient performed movements with the unaffected hand that, with the aid of manipulations in the VE, animated either the corresponding or contralateral virtual hand model (in real time). Our findings revealed that activations in the sensorimotor cortex of the affected hemisphere (the "inactive" cortex) were significantly increased simply by providing feedback of the contralateral hand. This effect was also evident in healthy subjects. In a follow up experiment, we mea- 
sured the MEPs in motor cortex [2], as healthy subjects were exposed to the same feedback conditions as in the fMRI study. Our data indicated that MEPs were substantially increased in both feedback conditions (corresponding and contralateral virtual hand models) but that the MEP amplitude increased by about $8 \%$ more in the contralateral relative to the corresponding feedback condition. This is in direct line with similar studies that have used a real, rather than a virtual, mirror feedback setup $[37,39,43]$ and adds to the body of evidence that suggests that sensorimotor training in VR may have similar effects on neural circuits to real-world training. The advantage of VR, however, is its versatility to allow more control over the type of feedback.

Studies on the use of non-VR presentations of visual stimulation support the possibility of this type of training. A study of horizontally flowing visual information on healthy persons that were stationary produced activation of the visual cortex and a corresponding decrease in the vestibular areas in a PET scan study by [28]. The authors postulated that this was a strategy to resolve the sensory conflict produced by these conditions. Brandt et al. showed activation of adjacent areas of the visual cortex and deactivation of multisensory vestibular centers in a PET scan study of healthy persons in response to large field optokinetic stimuli [32]. Similar findings were described by Konen et al. in an fMRI study of normal responses to optokinetic stimulation and pursuit/scanning type movements [70]. In an fMRI study of persons with chronic bilateral vestibular failure, subject's visual cortex activation was stronger in response to simulated visual motion than in healthy controls. The investigators describe this as an up-regulation of sensitivity to visual stimuli as a compensation for a lack of vestibular information [31]. Each of these studies support the use of visually simulated movement to elicit this functional activation/deactivation pattern when the brain is presented with conflicting information (simulated visual motion in a stationary subject).

\section{Visual feedback only in VR}

The preponderance of evidence for the therapeutic use of VR has come from intervention studies in the stroke patient population. The reason for this is in part attributed to the high prevalence of stroke and the particular challenges that upper extremity movement deficits pose to rehabilitation. Given the above, the following sections are weighted in reviewing VR applications for stroke populations, however, where data is available for other patient populations, we review those as well. Although the evidence generally supports VR's efficacy in retraining upper extremity (UE) function after stroke, the majority of these studies include case studies, small feasibility studies, or studies without strong control groups. Stroke rehabilitation training programs are most effective when requiring practice regimens that both engage and increasingly challenge the patient [13]. VR can aid in this sense by systematically adapting task difficulty to the patient's ability as he/she progresses through training and by providing a motivational factor to encourage longer engagement in the exercises than would otherwise be seen in a real-world environment [30].

Multiple authors describing the training of upper extremity reaching and functional activities in virtual environments have shown that motor skills can be learned through repetitive practice within both immersive and non-immersive and visually simple and complex virtual environments (see [49] for an extensive review). More recent studies have also shown similar results. Stewart et al. [114] describe a VR system that allows subjects to perform complex 3-dimensional tasks involving object manipulation and/or reaching. Following a twelve session intervention with this system, one of the two pilot subjects demonstrated improvements at the impairment and functional level. Piron and colleagues compared a group of subjects less than 3 months after a middle cerebral artery stroke [97]. Twenty-eight subjects performed upper extremity rehabilitation activities in a visual and auditory based, 2-dimensional virtual environment and a second group of 13 subjects performed a comparable volume of conventional upper extremity rehabilitation. The VR rehabilitation group made statistically significant gains on impairment (UE Fugl-Meyer) and functional independence measures (FIM) while the conventional rehabilitation group made smaller, non significant improvements in these measures.

These studies have focused on upper extremity training. Because of fiscal constraints, current service delivery models favor gait-training and proximal arm function [18]. However, the impact of even mild to moderate deficits in hand control effect many activities of daily living with detrimental consequences to social and work-related participation. In our own laboratory, a group of eight subjects with mild to moderate hemiparesis secondary to stroke performed 13 sessions of sensorimotor training in virtual environments that provided rich visual feedback as the subjects played 5 game-like activities targeting independent finger flexion, finger strength, and finger extension speed. Sub- 
jects improved in measures of independent finger flexion, finger speed, strength and range of motion measured during training tasks as well as in kinematic measures of reaching and grasping and clinical tests of upper extremity function. The Jebsen Test of Hand Function (JTHF) [56], a timed test of hand function and dexterity, was used to determine whether the kinematic improvements gained through practice in the VE measures transferred to real world functional activities. After training, the average time the subjects needed to complete the six subtests of the JTHF with their hemiplegic hand decreased significantly. In contrast, no changes were observed for the unaffected hand. The subjects' affected hand improved from pre-therapy to post-therapy on average by $12 \%$ [5].

We have recently developed a second generation of this system. The piano trainer is a refinement and elaboration of one of our previous simulations [81]. The new version consists of a complete virtual piano that plays the appropriate notes as they are pressed by the virtual fingers. The position and orientation of both hands as well as the flexion and abduction of the fingers are recorded in real time and translated into 3D movement of the virtual hands, shown on the screen in a first person perspective. The simulation can be utilized for training the hand alone to improve individuated finger movement (fractionation), or the hand and the arm together to improve the arm trajectory along with finger motion. This is achieved by manipulating the octaves on which the songs are played. These tasks can be done unilaterally or bilaterally. Other simulations provide practice in the integration of reach, hand-shaping and grasp using a pincer grip to catch and release a bird while it is perched on different objects located on different levels and sections of a 3D workspace [2-4], see also below, pp. 37-38.

In addition to upper extremity movement deficits after stroke, spatial neglect is another common syndrome following stroke, most frequently due to damage of the right hemisphere. Up to two-thirds of patients with acute right-hemisphere stroke demonstrate signs of contralesional neglect, failing to be aware of visual, auditory and or tactile stimuli coming from left of their midline in extrapersonal space. Hemispatial neglect has profound effects on the patient's ability to interact with and respond to their environments [64]. VR simulations have been employed with some success in several studies for both the assessment and treatment of visuo-spatial and visuo-motor neglect [55]. With manual exploration tasks, VR applications can detect small variations in performance undetectable by standard pa- per and pencil tests [16]. Training in VR has shown improvement in learning to cross a busy street, with left to right ratio scores (the ratio of objects seen on left to those seen on right) decreasing [23] and in reaching and grasping activities, where after training patients were able to code objects in the neglected space identically to those presented in their preserved space [9]. However, it was found that only patients without lesions in the inferior parietal/superior temporal regions benefited from this last training paradigm.

VR neglect intervention is not limited to ambulatory patients. Virtual environments have been used to assess spatial attention and neglect in wheelchair navigation. Here the subjects were asked to navigate a virtual path, encountering objects of varying complexity while in a wheelchair [21]. The VR navigation task was shown to have a strong correlation with the live wheelchair navigation task, and was able to detect deficits in mild patients. This implies VR shows promise as an efficient, sensitive measure of assessment and training for spatial neglect.

A patient's gait, or walking pattern, can be significantly altered after a stroke. Virtual realty (VR) offers a variety of methods to assess and improve several aspects of patient gait post-stroke. VR offers significant advantages over the traditional, qualitative, low intensity methods of physical therapy. VR enables the therapist to control duration, intensity, and feedback during specified treatment. The best VE is one that immerses and engages the subject in a realistic manner. To this end several modalities of human-computer interface have been employed [29]. Environments simulating both city and rural landscapes have been used for gait rehabilitation after stroke. These environments are used to retrain gait by providing visual cues to augment gait parameters such as stride length and walking velocity, as well as objects in the environment to augment obstacle avoidance. Walking speed is often severely reduced after stroke. Perception of the speed of one's environment has been shown to have an influence on the modulation of walking. Several VR studies have been conducted to quantify this effect. One study used VR to make continuous adjustments to the perception of optic flow speed [40]. Tests showed an inverse relationship between the VR optical flow speed and the walking speed, of patients after a stroke, though the correlation was weaker than that found in healthy subjects. In more recent studies, VE complexity of the city and rural landscapes has grown to include more life-like scenarios of street walking, collision avoidance and park strolling. In a small study, using scenar- 
ios of walking in a corridor, a park and across a street, and a motorized treadmill and a 6 dof motion platform, patients benefited from this practice by increasing their walking speed and adapting their gait to the changes in the terrain [41].

\subsection{Use of visual feedback in VR to treat Cerebral Palsy}

Children with Cerebral Palsy (CP) have difficulty controlling and coordinating voluntary muscle activity. In neuro-rehabilitation, these difficulties combined with the typical mentality of a child, can make this population challenging. Traditional therapies for muscle movement are repetitive and offer very little to keep a young mind occupied. Interactive VEs can provide a much wider array of activities and scenarios for muscle movement. In a selective motor control study of $\mathrm{CP}$ patients [17], children were asked to complete several ankle exercises using both video capture based training and conventional programs. While conventional therapy yielded more repetitions of the required exercises, the range of motion and hold time of stretch positions were greater in the VR group, thus the benefit of any movements was much greater during the VR exercises.

Approximately $50 \%$ of all children with CP sustain upper-extremity dysfunction to some degree [26]. VR's application extends well into this large area of neuromuscular rehabilitation. We have written above about the motivating advantages to VR. This obviously extends to UE exercises. A recently completed study was able to incorporate commercially available video games into their treatment regimen [26]. The study also revealed that to detect the full benefits of VR in a patient can require more sensitive diagnostic methods than are normally employed in physical and occupational therapy (e.g. Peabody Developmental Motor Scale, QUEST exam).

In addition to measurable changes in physical activity, VR has also shown promise in effecting neuroplasticity in CP patients. fMRI analysis, prior to VR training of the upper extremity of a child with hemiplegic $\mathrm{CP}$, showed predominately bilateral activation of the sensorimotor cortices and ipsilateral activation of the supplementary cortex. After training in a video capture-based VR system, this bilateral activation disappeared and the contralateral sensorimotor cortex was activated [130]. These recorded changes were closely associated with enhanced ability of the subject to perform reaching, dressing, and self-feeding tasks. VR's ability to create widely varying scenarios with a spectrum of difficulty also lends itself to gait training in CP patients [69].

\subsection{Use of visual feedback in VR for posture and balance rehabilitation}

The appropriate control of posture and balance underlies most functional skills and is achieved through timely integration of sensory information. For fall prevention, this integration requires rapid recalibration of visual, vestibular and somatosensory information. Disorders of the nervous system and aging lead to impairments in this mechanism. VR can be used in several ways to re-train postural control and balance. First, VR can be used to manipulate visual feedback to produce conflicts between visual, somatosensory and vestibular information as a way to train different sensory systems. Second, VR feedback can be systematically graded (in terms of speed and complexity) in order to challenge a person's static and dynamic postural control over the course of sensorimotor training.

Small sample investigations on the ability to manipulate visual stimuli in order to evoke conflict between visual, vestibular and somatosensory systems and corresponding changes in vestibular symptoms or postural responses have produced promising results. Scanning in complex visual environments can produce sensory conflict. Whitney et al. found that training in immersive VR may be useful for habituation activities for persons with visual/vestibular impairments. Using an immersive grocery store simulation, a subject with sub acute labyrinthine dysfunction experienced comparable symptoms to those she experienced in a real world grocery store. There was a correlation between the visual complexity of the simulation and her symptoms as well. Interestingly, a second subject with a more chronic lesion had adapted to this conflict and did not experience symptoms in this environment [125].

Immersive VR systems producing flow past a user's peripheral visual fields also produce a sense of motion similar to the optokinetic stimuli described by Brandt and Dieterich $[31,32]$. The perception of self motion this information creates can be manipulated in VE to elicit specific postural adjustments for training and rehabilitation purposes. In a study on healthy subjects visual stimuli that produced a conflict with simultaneous somatosensory and vestibular signals generated by horizontal motion elicited much stronger postural corrections measured by EMG than those produced by either horizontal motion or simulated visual motion alone [66]. In another study on twelve healthy subjects, center of pressure and perception of vertical measured with a wand in the subjects' hand was collected as subjects were presented with optic flow in three 
planes (yaw, pitch and roll). The effect of complexity of the visual flow patterns on postural response and perceived vertical was greatest in the roll plane and much less robust in pitch. Responses to varying levels and complexities of visual flow in the pitch plane varied significantly between subjects [65]. A third study by Keshner and colleagues described an increased effect of visually simulated motion on postural responses when subject base of support was decreased, making them more dependent on the erroneous, simulated visual information [116].

Mulavara et al. examined the responses of 30 healthy subjects to linear or rotating patterns of optic flow while walking straight on a treadmill. Subjects demonstrated adaptation to the condition of flow they were presented. Subjects displayed a consistent right bias on an eyes closed stepping task immediately following walking with a right rotating pattern of optic flow, and subjects presented with linear flow in the same plane and direction of their walking displayed no consistent bias [86]. These studies, illustrate the ability of immersive virtual environments to impact the integration of visual, vestibular and somatosensory inputs and subsequent postural responses. The incorporation of this element into rehabilitation programs with the goal of hastening the adaptation process in persons with vestibular pathology and to train postural responses in persons with balance impairments are the logical "next steps" for this line of inquiry.

Several authors discuss the use of balance training interventions using VR in a variety of populations. Oddsson et al. studied balance training in a tilted room environment simulated by lying on a surface that eliminated friction while being presented with virtually simulated immersive visual environments. Healthy subjects trained in the virtual environment made improvements in mediolateral critical time with eyes closed [90]. Training in VR allows for the safe and systematic training of sitting balance in persons with SCI. Kizony et al. studied the feasibility of applying VCVR technology for balance training in persons with paraplegia in a study with 13 subjects. Subjects utilized three 3D simulations, two that involved reaching for moving targets and a third that utilized trunk movement to control a snowboard. Users expressed that they enjoyed utilizing the equipment and reported high levels of presence during the activities. The subjects' scores on the simulations correlated well with their performance on a seated functional reaching task, suggesting that their real world balance and ability to perform the simulations measured a similar construct [67].
Several studies describe simple virtual rehabilitation interventions for persons with other neurologic pathologies. Fulk reported a case utilizing a VR-based balance intervention for a woman with MS. The subject performed a 12 week course of bodyweight supported ambulation training combined with non-immersive balance activities [38]. The subject improved her gait speed, endurance and standing balance measures. Thornton compared VR-based balance interventions and clinical balance activities in a group of patients with traumatic brain injuries. Two groups performed either activity based balance training or balance exercises in a 2 dimensional VR system. VR participants expressed higher degrees of enjoyment, made larger improvements on quantitative measures of balance and scored higher on balance confidence measures [121]. Pavlou et al. performed a comparison of conventional vestibular rehabilitation activities and exposure to visual vestibular conflict produced by an immersive VR system as part of a simulator based treatment that also included rotary chair and other whole body movement simulations. The VR / simulator treatment group made larger changes on posturography tests and larger improvements in symptoms intensity questionnaires than the conventional rehabilitation group [94].

The safety of VE-based balance training also makes it an effective tool for fall prevention interventions in elderly populations. VE can provide distracting environments or additional cognitive tasks, two conditions associated with increased frequency of falls in the elderly. Bisson and colleagues studied two groups of elderly subjects, one that trained on balance activities using visual biofeedback displaying force-plate data and a second performing juggling activities that required lateral reaches in a VCVR environment. Both groups achieved statistically significant improvements in reaction time, and the Community Balance and Mobility Scale [12].

\section{Integration of vision and haptics in VR}

A major development in the use of virtual environments has been the incorporation of tactile information and interaction forces into what was previously an essentially visual experience. Robots of varying complexity are being interfaced with more traditional VE presentations to provide haptic feedback that 1) enriches the sensory experience, 2) adds physical task parameters, and 3) provides forces that produce biomechanical and neuromuscular interactions with the virtual en- 
vironment that approximate real world movement more accurately than visual only VE's.

Simple haptic feedback can be utilized to add the perception of contact to skills like kicking a soccer ball or striking a piano key. Lam et al. describe a system that utilized vibratory discs to simulate the feeling of impact during this type of game. The authors cited advanced skill learning in a group of healthy subjects training with added tactile feedback [71]. Adamovich et al. used a force reflecting exoskeleton that simulates contact with piano keys [2]. Collisions with virtual world obstacles can also be used to teach normal movement trajectories such as the action required to place an object on a shelf [4] or step over a curb [124].

Some previous approaches utilized virtual tutors to model ideal trajectories [51,99]. Using haptic obstacles to indirectly shape trajectories may avoid the effects of the explicit, cognitive process associated with presenting a model, into what is usually an implicit process [14]. Further investigations into this potential advantage are necessary because of the significant increases in cost associated with adding haptic effects to virtual rehabilitation applications. Haptic environments can also exert global forces on the user such as antigravity support and viscous stabilization forces. This allows more disabled subjects to exercise reaching and object manipulation in 3D space, which invokes muscular force synergies that are typically used and, advertently, more appropriate neuromuscular feedback as well. Several authors employ these concepts in VR simulations designed to train reaching, grasping, and lifting. Wolbrecht et al. describe a haptic robotic interface that provides anti-gravity assistance as needed to lower functioning persons as they interact with virtual environments. They tested this approach on nine persons with chronic hemiparesis secondary to stroke. As a group these subjects improved on kinematic measures during robotic training and clinical measures of upper extremity function [127]. Our laboratory has investigated the feasibility of this "assist as needed" approach for the arm [4] as well as the hand [2]. Subjects in both studies performed extended training periods in a short period of time without adverse effects and made similar kinematic and real world functional improvements.

Other tasks involve contact and interaction with tools to achieve movement goals. In the real world, object manipulation produces an interaction between user and object that is unique (e.g. the angular momentum of the head of hammer). Haptics can simulate the interaction forces produced by tools in virtual environments. Lambercy et al. describe a haptic knob that can be applied to manipulate objects that vary in size and shape allowing for customization based on therapeutic goals [72]. Haptic forces can also be synchronized with visual feedback to improve a users' sense of agency in the virtual world. In two small studies involving healthy subjects, this feedback combination was found to be more effective for skill learning than visual only feedback in healthy subjects $[53,110]$. The distortion of forces in a virtual environment is another line of inquiry afforded by haptics. Patton et al. found that haptic forces that augmented the errors of subjects with strokes were more effective in teaching desired trajectories than haptic forces that guided subjects toward these trajectories. These effects were found in simple two dimensional VE [92] and an immersive three dimensional VE [91].

Our laboratory has developed a VR system that utilizes visual and haptic feedback for the sensorimotor training of the hemiparetic upper extremity, specifically to train arm reaching and hand manipulation in threedimensional space. For the upper arm training, each subject trained using 2 different, 3-dimensional virtually simulated reaching activities over the course of eight or nine sessions. Task one had subjects pick up cups off of a haptically rendered table and place them on haptic shelves. Collisions with the table shelves and other cups were solid, forcing subjects to alter their trajectory to complete the task. Task two required subjects to move through a standardized set of targets with no obstacles. In a group of four chronic stroke subjects [4], subjects demonstrated a $36 \%$ improvement in task duration, and a $45 \%$ improvement in hand trajectory smoothness on the task with no obstacles. The same subjects demonstrated a $42 \%$ reduction in task duration, and a $70 \%$ improvement in hand trajectory smoothness during the task that utilized haptic obstacles. These subjects seemed to respond to the independent condition of haptically rendered obstacles with more efficient learning. Future studies of this concept should include a larger sample, generalization testing and measurements of motor control.

For practice in hand manipulation, for patients with greater impairments, the piano trainer (see p. 34) can be combined with a force reflecting exoskeleton that can inhibit mass grasp patterns and/or provide for haptically rendered finger tip collisions. In a proof of concept study, three of our subjects utilized the CyberGrasp exoskeleton to facilitate extension of their inactive fingers while utilizing the virtual piano trainer for eight to nine, sixty to ninety-minute sessions. Each of these three subjects were in the chronic stage of their stroke 
recovery and were classified as level 5 hemiparesis for their arms and level three hemiparesis for their hands using the Chedoke McMaster Stroke Impairment Inventory [44]. Two of the three subjects made improvements in their scores on the Jebsen Test of Hand Function (by $13 \%$ and $11 \%$ ). It appears that further investigation of this approach for persons with moderate upper extremity hemiparesis is warranted.

It is controversial whether training the upper extremity as an integrated unit leads to better outcomes than training the proximal and distal components separately. Current rehabilitation practice describes the need to develop proximal control and mobility prior to initiating training of the hand. During recovery from a lesion the hand and arm are thought to compete with each other for neural territory. Therefore, emphasizing initial proximal training may actually have deleterious effects on the neuroplasticity and functional recovery of the hand. However, neural control mechanisms of arm transport and hand-object interaction are interdependent. Therefore, complex multisegmental motor training is thought to be more beneficial for skill retention. We used this system to examine the effectiveness of training the hand and arm as a functional unit. The virtual simulations used in this protocol include; 1) a three dimensional pinching task, (the arm transports the hand to the appropriate place to catch a flying bird and the fingers perform a pinching movement to place it on a tree), 2) a pong based game (the arm moves to control the paddle and finger extension engages the paddle allowing participants to compete with a live or computerized opponent, 3) a realistic full sized virtual piano keyboard and 4) a three-dimensional hammering game, in which the arm controls the position of the hammer in 3D space and finger flexion and extension controls the rotation of the hammer as it interacts with the target.

In an ongoing study, a group of 8 subjects with chronic strokes resulting in mild hemiparesis (mean Jebsen Test of Hand Function (JTHF) score $=152$ ), trained for three hours in each of 8 sessions over two weeks using these 4 simulations. Each subject demonstrated improvements in robotically collected kinematics but more importantly, the group demonstrated a mean improvement in JTHF of $21 \%(\mathrm{SD}=15 \%)$ and a corresponding improvement in Wolf Motor Function Test Aggregate Time of $24 \%$ (SD $=11 \%$ ) (unpublished observations). This data compares quite favorably to a study we published previously [5,81], in which we trained subjects using an earlier iteration of our system practicing tasks that emphasized finger movement only. Eight chronic subjects with a similar level of mild hemiparesis (JTHF pre-test of 140) performed a comparable volume of training resulting in a $10 \%$ improvement in JTHF time, approximately half of the improvements experienced by the subjects using our total training approach.

\subsection{Use of visual and haptic feedback in VR for gait rehabilitation}

Several interesting studies have been generated evaluating the integration of the LOKOMAT, a robotic gait orthosis and virtual environments. Wellner et al. describe a series of experiments manipulating point of view, haptic collisions and augmented auditory feedback with a group of healthy subjects as they step over virtual obstacles with a goal of developing an optimal training program for gait rehabilitation. Subjects in these experiments were more successful when provided with haptic feedback from collisions with the virtual obstacle, and with a lateral view of themselves and their obstacle during training. Furthermore, subjects expressed that auditory feedback that cued them regarding increased gait speed and the distance to approaching obstacles was helpful [124]. Tierney et al. describe the design of a system for the gait rehabilitation of persons with strokes utilizing a partial body weight support system. The authors propose that the normalization of gait speed afforded by BWS gait training, paired with semi-immersive virtual environments simulating realworld ambulation situations may provide more ecologically valid stimuli for gait rehabilitation (unpublished observations).

It is not clear which component of this system provides the positive effects - the robotic assistance or the VR. Mirelman et al. described an additive effect of VR simulations to robotic training for gait when compared to a similar volume of robot-only training [83]. This study compared two groups of subjects with strokes who performed ankle exercises utilizing the Rutgers Ankle, a six degrees of freedom robot. Nine subjects performed these activities in a VE. Nine more performed the same program receiving knowledge of results and performance feedback from a therapist. VE group subjects made larger improvements in gait speed, six minute walk test and community ambulation distance as measured by a pedometer. All of these comparisons reached statistical significance and were maintained at three month follow-up. Six of the nine subjects in the VE group made improvements in their gait velocity that were large enough to change their functional ambulation category as defined by Perry et al. [96]. 


\subsection{Use of visual and haptic feedback in VR to treat Cerebral Palsy}

A critical limitation of the vision-only VR technology for the CP population is the high degree of motor function required to access many formats of this technology. One approach to overcome this issue is the interfacing of virtual environments with robotic assistance to allow participation of more involved patients. To date, only a handful of small studies have utilized interactive haptic environments to train children with CP. Our laboratory has investigated the feasibility of the combination of robotically facilitated movements with rich VE and complex gaming applications. Qiu et al. describe the experience of two children with the NJIT-RVR system. One of the children made a $45^{\circ}$ improvement in active supination and the other subject demonstrated clinically significant improvements on the Melbourne Assessment of Upper Extremity Performance after training in VR for one hour per day, 3 days a week for three weeks [103].

One of the important assets of VE systems for the rehabilitation of children is their flexibility. Simple alterations to graphics and sound effects significantly improved time on task and attention levels in the children described above. For example, we have successfully developed and tested a reaching simulation where adults with strokes received adaptable robot assistance during reaching in three-dimensional space presented in stereo [4]. The same activity seemed to be not that interesting for $\mathrm{CP}$ children under 10. Adding simple sound and visual effects to the activity (simulating explosions of the target objects) was sufficient to substantially improve attention levels and compliance in this group of 8 children with hemiparetic CP [35]. This flexibility will allow therapists to tailor the presentation of complex interventions to the developmental and cognitive constraints presented by the diverse group of $\mathrm{CP}$ patients.

In a recent study, Fasoli et al. [34] describe a group of 5 to 12 year old children with UE hemiplegia secondary to $\mathrm{CP}$ performing 16 , sixty minute practice sessions in a simple virtual environment with assist as needed robotic facilitation over an eight week period. Each session, consisted of 640 repetitive, goal-directed planar reaching movements. Subjects demonstrated improvements in Quality of Upper Extremity Test and Upper Extremity Fugl-Meyer Assessment scores [34]. Finally, one study was able to use the LOKOMAT gait orthosis in conjunction with VE's to create a realistic haptic world designed to treat children with CP. Simu- lations utilizing this array included an obstacle course, wading in a stream, crossing a street and performing a virtual soccer activity. To date, proof concept studies performed on healthy subjects utilizing questionnaires have confirmed the realism of these simulations [69].

\section{Telerehabilitation}

Access to rehabilitation services in rural and other underserved areas is a critical healthcare issue. Telerehabilitation systems (TRS) are one of the approaches being developed to address this issue. Many TRS incorporate some form of VE in their presentation. Several authors have investigated upper extremity interventions utilizing TRS in pilot studies. Two studies examined the efficacy of TRS based interventions for the hemiparetic UE. In one study, impairment level and functional assessments approached statistically significant levels of improvement after TRS training of gross UE movements and finer grasping movements [51]. Carey et al. examined two groups performing finger exercise without direct supervision. The experimental group performed a tracking task presented via a TRS. Controls performed a home exercise program consisting of a similar volume of non-goal oriented finger movements. While clinical testing results were similar for the two groups, fMRI activation during a finger tracking task was higher in the TRS group after training. The results of this study indicate that even the simplest form of interactive visual feedback during sensorimotor training might be beneficial for facilitation of brain activation [22]. Heuser et al. utilized the Rutgers Master II, a haptic glove system, in a thirteen session telerehabilitation intervention that utilized VR simulations for 5 persons post surgery for carpal tunnel syndrome. Three of the five subjects made substantial strength gains measured clinically and all of the subjects expressed satisfaction with their telerehabilitation experience [50].

The studies above begin to establish effectiveness of TRS interventions but do not test for the independent condition of remote supervision. Deutsch et al. examined the effects of utilizing a TRS by having subjects with strokes perform ankle rehabilitation activities in a VE while supervised in person by a therapist. After three weeks of intervention the subjects performed the same protocol with remote supervision by a therapist. The subjects performance and the volume of activity performed during week four, the remote supervision week, was comparable to week three, suggesting that 
remote monitoring would not detract from the productivity of the session [29].

A study by Piron et al. compared 12 patients with stroke performing a remotely monitored telerehabilitation program for their hemiparetic UE, and another group of 12 subjects with stroke, performing a similar program of real-world UE activity in their homes supervised by a therapist. Both groups made comparable improvements in UE function and untrained reaching kinematics [98]. A second study by Piron et al. [100] compared two groups of 5 subjects with strokes, one that trained UE movements in a VE while supervised in person by a therapist and a second performing the same VE simulated training program and supervised by a therapist remotely, using video-conferencing equipment. The TRS group in this study made statistically significant improvements in motor performance while the in-person supervision group changes were not statistically significant [100]. While each of these studies cites comparable or superior benefits for TRS, these studies were small indicating a need for further study with larger numbers of patients.

The use of telerehabilitation is in the nascent stage of development and implementation. While the results of these small VR-based studies examining the clinical effectiveness of TRS are promising it is important to note that large studies that have evaluated the cost effectiveness, and practicality of implementation of telerehabilitation services in comparison to hospital based services have shown mixed results [61].

\section{Conclusions, limitations, and future directions}

Virtual reality technology may be an optimal tool for designing therapies that target neuroplastic mechanisms in the nervous system, allow for mass practice and provide training in complex environments that are sometimes impractical or impossible to create in the natural world. They also allow for access to rehabilitation services through telerehabilitation. Computerized systems are well suited to this and afford great precision in automatically adapting task difficulty based on individual subject's ever changing performance. When virtual reality simulations are interfaced with movement tracking and sensing glove systems they provide an engaging, motivating and adaptable environment where the motion of the limb displayed in the virtual world is a replication of the motion produced in the real world by the subject. Virtual environments can manipulate the specificity and frequency of visual and auditory feed- back, and can provide adaptive learning algorithms and graded rehabilitation activities that can be objectively and systematically manipulated to create individualized motor learning paradigms. Thus, it provides a rehabilitation tool that can be used to harness the nervous system's capacity for sensorimotor adaptation.

Virtual rehabilitation for movement disorders has been developing more slowly than virtual technologies in other areas of healthcare. In our opinion there are several factors underlying this trend. System development involves sophisticated interlacing between hardware and software which at the present time is expensive and requires considerable development expertise. The interdisciplinary nature of rehabilitation research also presents challenges. The design of interfaces to accommodate persons with impaired movement requires skills that span orthopedics, neuroscience, biomedical engineering, computer science and multiple rehabilitation disciplines. More studies are emerging to test VR's efficacy in rehabilitation, however, the effectiveness of these studies has not yet reached he higher levels of evidence found in large scale randomly controlled studies. The extent to which repetitive training offers neural and functional benefits beyond the novelty factor as well as the ability to integrate this form of therapy into a clinical setting remains unknown. Finally, and perhaps most important, the full potential of VR will only emerge after we gain a thorough understanding of how various sensory and haptic manipulations in VR affect neural processes. These issues should be a central focus of future investigations.

\section{Acknowledgements}

This work was supported in part by the National Institute on Disability and Rehabilitation Research, Rehabilitation Engineering Research Center Grant \# H133E050011.

\section{References}

[1] S. Adamovich, Q. Qiu, B. Talati, G. Fluet and A. Merians, Design of a virtual reality-based system for hand and arm rehabilitation, Proc International Conference on Rehabilitation Robotics, Noordwijk, The Netherlands, 2007, 958-964.

[2] S. Adamovich, G. Fluet, M. Mathai, Q. Qiu, J. Lewis and A. Merians, Design of a Complex Virtual Reality Simulation to Train Finger Motion for Persons with Hemiparesis: A feasibility study, J Neuroeng Rehabil (2009), in press. 
[3] S. Adamovich, G. Fluet, A. Merians, A. Mathai and Q. Qiu, Recovery of Hand Function in Virtual Reality: Training Hemiparetic Hand and Arm Together or Separately, 28th EMBC Annual International Conference Engineering in Medicine and Biology Society, Vancouver, Canada, 2008, 3475-3478.

[4] S. Adamovich, G. Fluet, Q. Qiu, A. Mathai and A. Merians, Incorporating haptic effects into three-dimensional virtual environments to train the hemiparetic upper extremity, IEEE Trans Neural Syst Rehabil Eng (2009), in press.

[5] S. Adamovich, A. Merians, R. Boian, M. Tremaine, G. Burdea, M. Recce et al., A virtual reality (VR)-based exercise system for hand rehabilitation post stroke, Presence $\mathbf{1 4}$ (2005), 161-174.

[6] S.V. Adamovich, K. August, A. Merians and E. Tunik, A virtual reality based system integrated with fMRI to study neural mechanisms underlying rehabilitation: a proof of concept study, Restor Neurol Neurosci 27(3) (2009), 209-223.

[7] E.L. Altschuler, S.B. Wisdom, L. Stone, C. Foster, D. Galasko, D.M. Llewellyn et al., Rehabilitation of hemiparesis after stroke with a mirror, Lancet 353(9169) (12 Jun 1999), 2035-2036.

[8] E.L. Altschuler, Interaction of vision and movement via a mirror, Perception 34(9) (2005), 1153-1155.

[9] C. Ansuini, A.C. Pierno, D. Lusher and U. Castiello, Virtual reality applications for the remapping of space in neglect patients, Restor Neurol Neurosci 24(4-6) (2006), 431-441.

[10] J. Bernhardt, N. Chitravas, I.L. Meslo, A.G. Thrift and B. Indredavik, Not all stroke units are the same: a comparison of physical activity patterns in Melbourne, Australia, and Trondheim, Norway, Stroke 39(7) (Jul 2008), 2059-2065.

[11] J. Bernhardt, H. Dewey, A. Thrift and G. Donnan, Inactive and alone: physical activity within the first 14 days of acute stroke unit care, Stroke 35(4) (Apr 2004), 1005-1009.

[12] Y. Bisson, B. Constant, H. Sveistrup and Y. Lajoie, Balance Training for the Elderly: Comparison between Virtual Reality and Visual Biofeedback, 6th World Conference on Aging and Physical Activity, 2004, London, 2004.

[13] D.T. Blake, F. Strata, R. Kempter and M.M. Merzenich, Experience-dependent plasticity in $\mathrm{S} 1$ caused by noncoincident inputs, J Neurophysiol 94(3) (Sep 2005), 2239-2250.

[14] L. Boyd and C. Winstein, Explicit information interferes with implicit motor learning of both continuous and discrete movement tasks after stroke, J Neurol Phys Ther 30(2) (Jun 2006), 46-57; discussion 8-9.

[15] S. Bray, S. Shimojo and J.P. O’Doherty, Direct instrumental conditioning of neural activity using functional magnetic resonance imaging-derived reward feedback, J Neurosci 27(28) (11 Jul 2007), 7498-7507.

[16] J. Broeren, M. Rydmark, A. Bjorkdahl and K.S. Sunnerhagen, Assessment and training in a 3-dimensional virtual environment with haptics: a report on 5 cases of motor rehabilitation in the chronic stage after stroke, Neurorehabil Neural Repair 21(2) (Jun 2007), 180-189.

[17] C. Bryanton, J. Bosse, M. Brien, J. McLean, A. McCormick and H. Sveistrup, Feasibility, motivation, and selective motor control: virtual reality compared to conventional home exercise in children with cerebral palsy, Cyberpsychol Behav 9(2) (Apr 2006), 123-128.

[18] G. Buccino, A. Solodkin and S.L. Small, Functions of the mirror neuron system: implications for neurorehabilitation, Cogn Behav Neurol 19(1) (Mar 2006), 55-63.

[19] G.C. Burdea, Virtual rehabilitation-benefits and challenges, Methods Inf Med 42(5) (2003), 519-523.
[20] A.J. Butler and S.J. Page, Mental practice with motor imagery: evidence for motor recovery and cortical reorganization after stroke, Arch Phys Med Rehabil 87(12 Suppl 2) (Dec 2006), S2-S11.

[21] L.J. Buxbaum, K.Y. Haaland, M. Hallett, L. Wheaton, K.M. Heilman, A. Rodriguez et al., Treatment of limb apraxia: moving forward to improved action, Am J Phys Med Rehabil 87(2) (Feb 2008), 149-161.

[22] J.R. Carey, W.K. Durfee, E. Bhatt, A. Nagpal, S.A. Weinstein, K.M. Anderson et al., Comparison of finger tracking versus simple movement training via telerehabilitation to alter hand function and cortical reorganization after stroke, Neurorehabil Neural Repair 21(3) (May-Jun 2007), 216-232.

[23] J.R. Carey, C.D. Evans, D.C. Anderson, E. Bhatt, A. Nagpal, T.J. Kimberley et al., Safety of 6-Hz Primed Low-Frequency rTMS in Stroke, Neurorehabil Neural Repair (17 Sep 2007).

[24] J.R. Carey, T.J. Kimberley, S.M. Lewis, E.J. Auerbach, L. Dorsey, P. Rundquist et al., Analysis of fMRI and finger tracking training in subjects with chronic stroke, Brain $125(\mathrm{Pt}$ 4) (Apr 2002), 773-788.

[25] P. Celnik, K. Stefan, F. Hummel, J. Duque, J. Classen and L.G. Cohen, Encoding a motor memory in the older adult by action observation, Neuroimage 29(2) (15 Jan 2006), 677684.

[26] Y.P. Chen, L.J. Kang, T.Y. Chuang, J.L. Doong, S.J. Lee, M.W. Tsai et al., Use of virtual reality to improve upperextremity control in children with cerebral palsy: a singlesubject design, Phys Ther 87(11) (Nov 2007), 1441-1457.

[27] S. Coote, B. Murphy, W. Harwin and E. Stokes, The effect of the GENTLE/s robot-mediated therapy system on arm function after stroke, Clin Rehabil 22(5) (May 2008), 395405.

[28] B.M. de Jong, S. Shipp, B. Skidmore, R.S. Frackowiak and S. Zeki, The cerebral activity related to the visual perception of forward motion in depth, Brain 117(Pt 5) (Oct 1994), 1039-1054

[29] J.E. Deutsch, J.A. Lewis and G. Burdea, Technical and patient performance using a virtual reality-integrated telerehabilitation system: preliminary finding, IEEE Trans Neural Syst Rehabil Eng 15(1) (Mar 2007), 30-35.

[30] J.E. Deutsch, A.S. Merians, S. Adamovich, H. Poizner and G.C. Burdea, Development and application of virtual reality technology to improve hand use and gait of individuals poststroke, Restor Neurol Neurosci 22(3-5) (2004), 371-386.

[31] M. Dieterich, T. Bauermann, C. Best, P. Stoeter and P. Schlindwein, Evidence for cortical visual substitution of chronic bilateral vestibular failure (an fMRI study), Brain 130(Pt 8) (Aug 2007), 2108-2116.

[32] M. Dieterich, S.F. Bucher, K.C. Seelos and T. Brandt, Horizontal or vertical optokinetic stimulation activates visual motion-sensitive, ocular motor and vestibular cortex areas with right hemispheric dominance, An fMRI study, Brain 121(Pt 8) (Aug 1998), 1479-1495.

[33] A.Y. Dvorkin, M. Shahar and P.L. Weiss, Reaching within video-capture virtual reality: using virtual reality as a motor control paradigm, Cyberpsychol Behav 9(2) (Apr 2006), 133-136.

[34] S.E. Fasoli, M. Fragala-Pinkham, R. Hughes, N. Hogan, H.I. Krebs and J. Stein, Upper Limb Robotic Therapy for Children with Hemiplegia, Am J Phys Med Rehabil 87(11) (Nov 2008), 929-936.

[35] G.G. Fluet, Q. Qiu, S. Saleh, D. Ramirez, S. Adamovich, D. Kelly and H. Parikh, Robot-Assisted Virtual Rehabilitation (NJIT-RAVR) system for children with upper extremity 
hemiplegia, 2009 International Workshop on Virtual Rehabilitation, 2009, in press.

[36] K.M. Friel, S. Barbay, S.B. Frost, E.J. Plautz, A.M. Stowe, N. Dancause et al., Effects of a rostral motor cortex lesion on primary motor cortex hand representation topography in primates, Neurorehabil Neural Repair 21(1) (Jan-Feb 2007), 51-61.

[37] K. Fukumura, K. Sugawara, S. Tanabe, J. Ushiba and Y. Tomita, Influence of mirror therapy on human motor cortex, Int J Neurosci 117(7) (Jul 2007), 1039-14048.

[38] G.D. Fulk, Locomotor training and virtual reality-based balance training for an individual with multiple sclerosis: a case report, J Neurol Phys Ther 29(1) (Mar 2005), 34-42.

[39] K. Funase, T. Tabira, T. Higashi, N. Liang and T. Kasai, Increased corticospinal excitability during direct observation of self-movement and indirect observation with a mirror box, Neurosci Lett 419(2) (29 May 2007), 108-112.

[40] J. Fung, F. Malouin, B.J. McFadyen BJ, Comeau F, Lamontagne A, Chapdelaine $\mathrm{S}$, et al. Locomotor rehabilitation in a complex virtual environment. Conf Proc IEEE Eng Med Biol Soc. 2004;7:4859-61.

[41] J. Fung, C.L. Richards, F. Malouin, B.J. McFadyen and A. Lamontagne, A treadmill and motion coupled virtual reality system for gait training post-stroke, Cyberpsychol Behav $\mathbf{9}(2)$ (Apr 2006), 157-162.

[42] A. Gaggioli, A. Meneghini, F. Morganti, M. Alcaniz and G. Riva, A strategy for computer-assisted mental practice in stroke rehabilitation, Neurorehabil Neural Repair 20(4) (Dec 2006), 503-507.

[43] M.I. Garry, A. Loftus and J.J. Summers, Mirror, mirror on the wall: viewing a mirror reflection of unilateral hand movements facilitates ipsilateral M1 excitability, Exp Brain Res 163(1) (May 2005), 118-122.

[44] C. Gowland, P. Stratford, M. Ward, J. Moreland, W. Torresin, S. Van Hullenaar et al., Measuring physical impairment and disability with the Chedoke-McMaster Stroke Assessment, Stroke 24(1) (Jan 1993), 58-63.

[45] M.S. Graziano and C.G. Gross, Spatial maps for the control of movement, Curr Opin Neurobiol 8(2) (Apr 1998), 195201.

[46] M.S. Graziano and C.G. Gross, Visual responses with and without fixation: neurons in premotor cortex encode spatial locations independently of eye position, Exp Brain Res 118(3) (Feb 1998), 373-380.

[47] M.S. Graziano, Where is my arm? The relative role of vision and proprioception in the neuronal representation of limb position, Proc Nat Acad Sci 96(18) (31 Aug 1999), 1041810421.

[48] A. Hadipour-Niktarash, C.K. Lee, J.E. Desmond and R. Shadmehr, Impairment of retention but not acquisition of a visuomotor skill through time-dependent disruption of primary motor cortex, J Neurosci 27(49) (5 Dec 2007), 1341313419.

[49] A. Henderson, N. Korner-Bitensky and M. Levin, Virtual reality in stroke rehabilitation: a systematic review of its effectiveness for upper limb motor recovery, Top Stroke Rehabil 14(2) (Mar-Apr 2007), 52-61.

[50] A. Heuser, H. Kourtev, S. Winter, D. Fensterheim, G. Burdea, V. Hentz et al., Telerehabilitation using the Rutgers Master II glove following carpal tunnel release surgery: proof-ofconcept, IEEE Trans Neural Syst Rehabil Eng 15(1) (Mar 2007), 43-49.

[51] M.K. Holden, T.A. Dyar and L. Dayan-Cimadoro, Telerehabilitation using a virtual environment improves upper ex- tremity function in patients with stroke, IEEE Trans Neural Syst Rehabil Eng 15(1) (Mar 2007), 36-42.

[52] M.K. Holden, Virtual environments for motor rehabilitation: review, Cyberpsychol Behav 8(3) (Jun 2005), 187-211; discussion 2-9.

[53] F.C. Huang, R.B. Gillespie and A.D. Kuo, Visual and haptic feedback contribute to tuning and online control during object manipulation, J Motor Behav 39(3) (May 2007), 179-193.

[54] S.H. Jang, S.H. You, M. Hallett, Y.W. Cho, C.M. Park, S.H. Cho et al., Cortical reorganization and associated functional motor recovery after virtual reality in patients with chronic stroke: an experimenter-blind preliminary study, Arch Phys Med Rehabil 86(11) (Nov 2005), 2218-2223.

[55] M.J. Jannink, M. Aznar, A.C. de Kort, W. van de Vis, P. Veltink and H. van der Kooij, Assessment of visuospatial neglect in stroke patients using virtual reality: a pilot study, Int J Rehabil Res (7 Aug 2008).

[56] R.H. Jebsen, N. Taylor, R.B. Trieschmann, M.J. Trotter and L.A. Howard, An objective and standardized test of hand function, Arch Phys Med Rehabil 50(6) (Jun 1969), 311-319.

[57] W.M. Jenkins and M.M. Merzenich, Reorganization of neocortical representations after brain injury: a neurophysiological model of the bases of recovery from stroke, Progr Brain Res 71 (1987), 249-266.

[58] A.M. Jette, The post-stroke rehabilitation outcomes project, Arch Phys Med Rehabil 86(12 Suppl 2) (Dec 2005), S124S1S5.

[59] D.U. Jette, N.K. Latham, R.J. Smout, J. Gassaway, M.D. Slavin and S.D. Horn, Physical therapy interventions for patients with stroke in inpatient rehabilitation facilities, Phys Ther 85(3) (Mar 2005), 238-248.

[60] D.U. Jette, R.L. Warren and C. Wirtalla, The relation between therapy intensity and outcomes of rehabilitation in skilled nursing facilities, Arch Phys Med Rehabil 86(3) (Mar 2005), 373-379.

[61] D. Kairy, P. Lehoux, C. Vincent and M. Visintin, A systematic review of clinical outcomes, clinical process, healthcare utilization and costs associated with telerehabilitation, Disabil Rehabil (7 Jul 2008), 1-21.

[62] S. Kakei, D.S. Hoffman and P.L. Strick, Sensorimotor transformations in cortical motor areas, Neurosci Res 46(1) (May 2003), 1-10.

[63] R.V. Kenyon, J. Leigh and E.A. Keshner, Considerations for the future development of virtual technology as a rehabilitation tool, J Neuroeng Rehabil 1(1) (23 Dec 2004), 13.

[64] G. Kerkhoff and Y. Rossetti, Plasticity in spatial neglect: recovery and rehabilitation, Restor Neurol Neurosci 24(4-6) (2006), 201-206.

[65] E.A. Keshner, K. Dokka and R.V. Kenyon, Influences of the perception of self-motion on postural parameters, Cyberpsychol Behav 9(2) (Apr 2006), 163-166.

[66] E.A. Keshner, R.V. Kenyon and J. Langston, Postural responses exhibit multisensory dependencies with discordant visual and support surface motion, J Vestib Res 14(4) (2004), 307-319.

[67] R. Kizony, L. Raz, N. Katz, H. Weingarden and P.L. Weiss, Video-capture virtual reality system for patients with paraplegic spinal cord injury, J Rehabil Res Dev 42(5) (Sep-Oct 2005), 595-608.

[68] J.A. Kleim, S. Barbay, N.R. Cooper, T.M. Hogg, C.N. Reidel, M.S. Remple et al., Motor learning-dependent synaptogenesis is localized to functionally reorganized motor cortex, Neurobiol Learn Mem 77(1) (Jan 2002), 63-77. 
[69] A. Koenig, M. Wellner, S. Koneke, A. Meyer-Heim, L. Lunenburger and R. Riener, Virtual gait training for children with cerebral palsy using the Lokomat gait orthosis, Stud Health Technol Inform 132 (2008), 204-209.

[70] C.S. Konen, R. Kleiser, R.J. Seitz and F. Bremmer, An fMRI study of optokinetic nystagmus and smooth-pursuit eye movements in humans, Exp Brain Res 165(2) (Aug 2005), 203-216.

[71] P. Lam, D. Hebert, J. Boger, H. Lacheray, D. Gardner, J. Apkarian et al., A haptic-robotic platform for upper-limb reaching stroke therapy: preliminary design and evaluation results, J Neuroeng Rehabil 5 (2008), 15.

[72] O. Lambercy, L. Dovat, R. Gassert, E. Burdet, C.L. Teo and T. Milner, A haptic knob for rehabilitation of hand function, IEEE Trans Neural Syst Rehabil Eng 15(3) (Sep 2007), 356366.

[73] A. Lamontagne, J. Fung, B.J. McFadyen and J. Faubert, Modulation of walking speed by changing optic flow in persons with stroke, J Neuroeng Rehabil 4 (2007), 22.

[74] C. Lang, J. Macdonald and C. Gnip, Counting repetitions: an observational study of outpatient therapy for people with hemiparesis post-stroke, J Neurol Phys Ther 31(1) (Mar 2007), 3-11.

[75] G. Leonard and F. Tremblay, Corticomotor facilitation associated with observation, imagery and imitation of hand actions: a comparative study in young and old adults, Exp Brain Res 177(2) (Feb 2007), 167-175.

[76] J.W. Lewis and D.C. Van Essen, Corticocortical connections of visual, sensorimotor, and multimodal processing areas in the parietal lobe of the macaque monkey, J Comp Neurol 428(1) (4 Dec 2000), 112-137.

[77] S.J. Lewis, A. Slabosz, T.W. Robbins, R.A. Barker and A.M. Owen, Dopaminergic basis for deficits in working memory but not attentional set-shifting in Parkinson's disease, $\mathrm{Neu}$ ropsychologia 43(6) (2005), 823-832.

[78] J. Liepert, H. Bauder, H.R. Wolfgang, W.H. Miltner, E. Taub and C. Weiller, Treatment-induced cortical reorganization after stroke in humans, Stroke 31(6) (Jun 2000), 1210-1216.

[79] H.W. Mahncke, A. Bronstone and M.M. Merzenich, Brain plasticity and functional losses in the aged: scientific bases for a novel intervention, Progress Brain Res 157 (2006), 81109.

[80] R. McCloy and R. Stone, Science, medicine, and the future. Virtual reality in surgery, BMJ 323(7318) (20 Oct 2001), 912-915.

[81] A.S. Merians, H. Poizner, R. Boian, G. Burdea and S. Adamovich, Sensorimotor training in a virtual reality environment: does it improve functional recovery poststroke? Neurorehabil Neural Repair 20(2) (Jun 2006), 252-267.

[82] A.S. Merians, E. Tunik and S.V. Adamovich, Virtual Reality to Maximize Function for Hand and Arm Rehabilitation: Exploration of Neural Mechanisms, in: Innovation in Rehabilitation Technology, A. Gaggioli, ed., IOS Press 2009, pp. 109-125.

[83] A. Mirelman, P. Bonato and J.E. Deutsch, Effects of training with a robot-virtual reality system compared with a robot alone on the gait of individuals after stroke, Stroke 40 (2009), 169-174.

[84] W. Muellbacher, B. Boroojerdi, U. Ziemann and M. Hallett, Analogous corticocortical inhibition and facilitation in ipsilateral and contralateral human motor cortex representations of the tongue, J Clin Neurophysiol 18(6) (Nov 2001), $550-558$.
[85] W. Muellbacher, U. Ziemann, B. Boroojerdi, L. Cohen and M. Hallett, Role of the human motor cortex in rapid motor learning, Exp Brain Res 136(4) (Feb 2001), 431-438.

[86] A.P. Mulavara, J.T. Richards, T. Ruttley, A. Marshburn, Y. Nomura and J.J. Bloomberg, Exposure to a rotating virtual environment during treadmill locomotion causes adaptation in heading direction, Exp Brain Res 166(2) (Oct 2005), 210219.

[87] R.J. Nudo, G.W. Milliken, W.M. Jenkins and M.M. Merzenich, Use-dependent alterations of movement representations in primary motor cortex of adult squirrel monkeys, J Neurosci 16(2) (15 Jan 1996), 785-807.

[88] R.J. Nudo and G.W. Milliken, Reorganization of movement representations in primary motor cortex following focal ischemic infarcts in adult squirrel monkeys, J Neurophysiol 75(5) (May 1996), 2144-2149.

[89] R.J. Nudo, E.J. Plautz and S.B. Frost, Role of adaptive plasticity in recovery of function after damage to motor cortex, Muscle Nerve 24(8) (Aug 2001), 1000-10019.

[90] L.I. Oddsson, R. Karlsson, J. Konrad, S. Ince, S.R. Williams and E. Zemkova, A rehabilitation tool for functional balance using altered gravity and virtual reality, J Neuroeng Rehabil 4 (2007), 25.

[91] J.L. Patton, M. Kovic and F.A. Mussa-Ivaldi, Customdesigned haptic training for restoring reaching ability to individuals with poststroke hemiparesis, J Rehabil Res Dev 43(5) (Aug-Sep 2006), 643-656.

[92] J.L. Patton, M.E. Stoykov, M. Kovic and F.A. Mussa-Ivaldi, Evaluation of robotic training forces that either enhance or reduce error in chronic hemiparetic stroke survivors, Exp Brain Res 168(3) (Jan 2006), 368-383.

[93] S. Patuzzo, A. Fiaschi and P. Manganotti, Modulation of motor cortex excitability in the left hemisphere during action observation: a single- and paired-pulse transcranial magnetic stimulation study of self- and non-self-action observation, Neuropsychologia 41(9) (2003), 1272-1278.

[94] M. Pavlou, A. Lingeswaran, R.A. Davies, M.A. Gresty and A.M. Bronstein, Simulator based rehabilitation in refractory dizziness, J Neurol 251(8) (Aug 2004), 983-995.

[95] D. Perani, F. Fazio, N.A. Borghese, M. Tettamanti, S. Ferrari, J. Decety et al., Different brain correlates for watching real and virtual hand actions, Neuroimage 14(3) (Sep 2001), 749758.

[96] J. Perry, M. Garrett, J.K. Gronley and S.J. Mulroy, Classification of walking handicap in the stroke population, Stroke 26(6) (Jun 1995), 982-989.

[97] L. Piron, P. Tombolini, P. Turolla, C. Zucconi, M. Agostini, M. Dam et al., Reinforced feedback in virtual environment facilitates the arm motor recovery in patients after a recent stroke, Virtual Rehabil 2007 (2007), 121-123.

[98] L. Piron, P. Tonin, F. Cortese, M. Zamploni, F. Piccione, M. Agostini et al., Post stroke arm telerehabilitation webbased, 2006 International Workshop on Virtual Rehabilitation (2006), 145-148.

[99] L. Piron, P. Tonin, F. Piccione, V. Iaia, E. Trevello and M. Dam, Virtual environment training for arm motor rehabilitation, Presence 16(6) (2005), 732-740.

[100] L. Piron, A. Turolla, P. Tonin, F. Piccione, L. Lain and M. Dam, Satisfaction with care in post-stroke patients undergoing a telerehabilitation programme at home, $J$ Telemed Telecare 14(5) (2008), 257-260.

[101] E.J.Plautz, G.W. Milliken and R.J. Nudo, Effects of repetitive motor training on movement representations in adult squirrel 
monkeys: role of use versus learning, Neurobiol Learn Mem 74(1) (Jul 2000), 27-55.

[102] M.B. Powers and P.M. Emmelkamp, Virtual reality exposure therapy for anxiety disorders: A meta-analysis, J Anxiety Disord 22(3) (2008), 561-569.

[103] Q. Qiu, S. Saleh, D. Ramirez, G.G. Fluet, H. Parikh, D. Kelly et al., The New Jersey Institute of Technology robotassisted virtual rehabilitation (NJIT-RVR) systemfor chidren with cerebral palsy: a feasibility study, J Neuroeng Rehabil (2009), in press.

[104] A.G. Richardson, S.A. Overduin, A. Valero-Cabre, C. PadoaSchioppa, A. Pascual-Leone, E. Bizzi et al., Disruption of primary motor cortex before learning impairs memory of movement dynamics, J Neurosci 26(48) (29 Nov 2006), 1246612470.

[105] G. Riva, G. Castelnuovo and F. Mantovani, Transformation of flow in rehabilitation: the role of advanced communication technologies, Behav Res Methods 38(2) (May 2006), 237244.

[106] G. Riva, Virtual environments in neuroscience, IEEE Trans Inf Technol Biomed 2(4) (Dec 1998), 275-281.

[107] A. Rizzo and G. Kim, A SWOT analysis of the field of virtual reality rehabilitation and therapy, Presence 14(2) (2005), 2005.

[108] A.A. Rizzo, K. Graap, K. Perlman, R.N. McLay, B.O. Rothbaum, G. Reger et al., Virtual Iraq: initial results from a VR exposure therapy application for combat-related PTSD, Stud Health Technol Inform 132 (2008), 420-425.

[109] I. Salimi, K.M. Friel and J.H. Martin, Pyramidal tract stimulation restores normal corticospinal tract connections and visuomotor skill after early postnatal motor cortex activity blockade, J Neurosci 28(29) (16 Jul 2008), 7426-7434.

[110] R.B. Singapogu, S.T. Sander, T.C. Burg and W.S. Cobb, Comparative study of haptic training versus visual training for kinesthetic navigation tasks, Studies Health Technol Informatics 132 (2008), 469-471.

[111] S.L. Small, P. Hlustik, D.C. Noll, C. Genovese and A. Solodkin, Cerebellar hemispheric activation ipsilateral to the paretic hand correlates with functional recovery after stroke, Brain 125(Pt 7) (Jul 2002), 1544-1557.

[112] K. Stefan, L.G. Cohen, J. Duque, R. Mazzocchio, P. Celnik, L. Sawaki et al., Formation of a motor memory by action observation, J Neurosci 25(41) (12 Oct 2005), 9339-9346.

[113] I. Stepniewska, P.C. Fang and J.H. Kaas, Microstimulation reveals specialized subregions for different complex movements in posterior parietal cortex of prosimian galagos, Proc Nat Acad 102(13) (29 Mar 2005), 4878-4883.

[114] J.C. Stewart, S.C. Yeh, Y. Jung, H. Yoon, M. Whitford, S.Y. Chen et al., Intervention to enhance skilled arm and hand movements after stroke: A feasibility study using a new virtual reality system, J Neuroeng Rehabil 4 (2007), 21.

[115] A.P. Strafella and T. Paus, Modulation of cortical excitability during action observation: a transcranial magnetic stimulation study, Neuroreport 11(10) (14 Jul 2000), 2289-2292.

[116] J.W. Streepey, R.V. Kenyon and E.A. Keshner, Visual motion combined with base of support width reveals variable field dependency in healthy young adults, Exp Brain Res 176(1) (Jan 2007), 182-187.

[117] H. Sveistrup, Motor rehabilitation using virtual reality, $J$ Neuroeng Rehabil 1(1) (10 Dec 2004), 10.

[118] E. Taub, N. Miller, T. Novack, E. Cook, W. Fleming, C. Nepomuceno et al., Technique to improve chronic motor deficit after stroke, Arch Phys Med Rehabil 74 (1993), 347354.

[119] E. Taub, G. Uswatte and R. Pidikiti, Constraint-Induced Movement Therapy: a new family of techniques with broad application to physical rehabilitation - a clinical review, $J$ Rehab Res Dev 36(3) (Jul 1999), 237-251.

[120] E. Taub and S.L. Wolf, Constraint induced movement techniques to facilitate upper extremity use in stroke patients, Top Stroke Rehabil 3 (1997), 38-61.

[121] M. Thornton, S. Marshall, J. McComas, H. Finestone, A. McCormick and $\mathrm{H}$. Sveistrup, Benefits of activity and virtual reality based balance exercise programmes for adults with traumatic brain injury: perceptions of participants and their caregivers, Brain Inj 19(12) (Nov 2005), 989-1000.

[122] A. Viau, A.G. Feldman, B.J. McFadyen and M.F. Levin, Reaching in reality and virtual reality: a comparison of movement kinematics in healthy subjects and in adults with hemiparesis, J Neuroeng Rehabil 1(1) (14 Dec 2004), 11.

[123] P.L. Weiss, D. Rand, N. Katz and R. Kizony, Video capture virtual reality as a flexible and effective rehabilitation tool, $J$ Neuroeng Rehabil 1(1) (20 Dec 2004), 12.

[124] M. Wellner, T. Thuring, E. Smajic, J. von Zitzewitz, A. Duschau-Wicke and R. Riener, Obstacle crossing in a virtual environment with the rehabilitation gait robot LOKOMAT, Studies Health Technol Informatics 125 (2007), 497-499.

[125] S.L. Whitney, P.J. Sparto, L.F. Hodges, S.V. Babu, J.M. Furman and M.S. Redfern, Responses to a virtual reality grocery store in persons with and without vestibular dysfunction, Cyberpsychol Behav 9(2) (Apr 2006), 152-156.

[126] S.P. Wise, S.L. Moody, K.J. Blomstrom and A.R. Mitz, Changes in motor cortical activity during visuomotor adaptation, Exp Brain Res 121(3) (Aug 1998), 285-299.

[127] E.T. Wolbrecht, V. Chan, D.J. Reinkensmeyer and J.E. Bobrow, Optimizing compliant, model-based robotic assistance to promote neurorehabilitation, IEEE Trans Neural Syst Rehabil Eng 16(3) (Jun 2008), 286-297.

[128] S. Wolf, D. LeCraw, L. Barton and B. Jann, Forced use of hemiplegic upper extremities to reverse the effect of learned non-use among chronic stroke and head injured patients, Exp Neurol 104 (1989), 125-132.

[129] S.H. You, S.H. Jang, Y.H. Kim, M. Hallett, S.H. Ahn, Y.H. Kwon et al., Virtual reality-induced cortical reorganization and associated locomotor recovery in chronic stroke: an experimenter-blind randomized study, Stroke 36(6) (Jun 2005), 1166-1171.

[130] S.H. You, S.H. Jang, Y.H. Kim, Y.H. Kwon, I. Barrow and M. Hallett, Cortical reorganization induced by virtual reality therapy in a child with hemiparetic cerebral palsy, Dev Med Child Neurol 47(9) (Sep 2005), 628-635. 